Revista de Metalurgia 52(4)

Octubre-Diciembre 2016, e081

ISSN-L: 0034-8570

doi: http://dx.doi.org/10.3989/revmetalm.081

\title{
Aceros sinterizados al Mo con gradiente funcional
}

\author{
Manuel Cisneros-Belmonte, ${ }^{\bowtie}$ José M. Ruiz-Román, Luis E. García-Cambronero \\ E.T.S. de Ingenieros de Minas y Energía, Universidad Politécnica de \\ Madrid, C/Ríos Rosas, 21, 28003 Madrid, España \\ Autor para la correspondencia: manuel.cisneros@upm.es
}

Enviado: 23 Octubre 2015; Aceptado: 2 Noviembre 2016; Publicado on-Line: 19 Diciembre 2016

RESUMEN: Los materiales con gradiente funcional (FGM), los multimateriales, tratan de satisfacer los numerosos requerimientos demandados a las piezas con una combinación determinada de composiciones y microestructuras. La compatibilidad necesaria entre materiales condiciona el proceso de fabricación y el logro de una interfase, no siempre difusa. La metalurgia de polvos es una de las técnicas empleadas en la fabricación de materiales con gradiente funcional y, en concreto la compactación en matriz una de las posibles técnicas de conformado de estos materiales. En el presente trabajo se propone un proceso de conformado de un acero con gradiente funcional que varía su composición entre un acero con alto contenido en molibdeno y cobre y otro acero con cobre, sin molibdeno, con el objeto de concentrar este elemento en la superficie de la pieza, incrementando las propiedades mecánicas. El estudio se completa con la evaluación de propiedades físicas (densidad y distribución de porosidad), propiedades mecánicas (dureza, resistencia a tracción y alargamiento) y análisis microestructural mediante microscopía óptica y electrónica de barrido.

PALABRAS CLAVE: Acero al molibdeno; Gradiente funcional; Nitrógeno; Propiedades mecánicas; Sinterización

Citation / Cómo citar este artículo: Cisneros-Belmonte, M., Ruiz-Román, J.M., Cambronero, L.E.G. (2016) "Aceros sinterizados al Mo con gradiente funcional”. Rev. Metal. 52(4):e081. doi: http://dx.doi.org/10.3989/revmetalm.081

ABSTRACT: Functionally Graded Mo sintered steels. Functionally graded materials (FGM), the multimaterials, strive to satisfy the numerous requirements demanded of parts in a given combination of compositions and microstructures. The required material compatibility lead the manufacturing process and the achieving of an interface, not always diffuse. Powder metallurgy is one of the techniques used in manufacturing functionally graded materials, in particular the compaction matrix of the possible techniques for forming these materials. In this paper, a process of forming a functionally graded steel based on the use of a high molybdenum steel with cooper and other steel with copper, without molybdenum, is proposed with the aim of concentrating this element to the surface of the workpiece, increasing the mechanical strength. The study is completed with the evaluation of physical properties (density and porosity distribution), mechanical properties (hardness, tensile strength and elongation) and microstructural analysis by optical and scanning electron microscopy.

KEYWORDS: Functionally graded; Mechanical properties; Molybdenum alloyed steel; Nitrogen; Sintering

Copyright: (C) 2016 CSIC. Este es un artículo de acceso abierto distribuido bajo los términos de la licencia Creative Commons Attribution (CC BY) España 3.0. 


\section{INTRODUCCIÓN}

Los materiales con gradiente funcional, y los materiales multifuncionales, se distinguen por ofrecer un amplio abanico de propiedades correspondientes a la microestructura y composición de las partes que los componen. Son una integración de materiales ligados por una interfase, no siempre difusa, como respuesta a los requerimientos (mecánicos, térmicos, eléctricos, resistencia a la corrosión, etc.) de los componentes en cada punto (Thomazic et al., 2010).

Existen numerosos casos de materiales naturales con gradiente funcional como son los huesos, los dientes, el bambú, etc., a diferencia de los materiales compuestos no supone una matriz que envuelva un refuerzo. Cada punto o zona de la pieza tiene unas propiedades diferentes; así, superficies sometidas a desgaste pueden ser materiales resistente a la abrasión como las cerámicas y el interior de la pieza esté fabricado con material con mayor tenacidad como puede ser un metal.

El origen del término materiales con gradiente funcional o FGM (functionally graded materials) se sitúa en Japón a mediados de los 80 del siglo XX, en el inicio de las investigaciones en materiales para la industria aeroespacial, dando forma una combinación de propiedades con materiales refractarios tenaces y tratando de evitar las tensiones y deformaciones que suceden en materiales disimilares mediante una transición gradual de la composición. A este programa le siguieron en otros países como USA, Alemania, China, Suiza, ... más recursos en la misma línea de investigación. El crecimiento de las investigaciones en materiales con gradiente funcional ha sido de los mayores en materiales estructurales (Mortensen y Suresh, 1995). Las posibilidades de estas combinaciones se trasladaron a otras aplicaciones no estructurales tratando de optimizar sus propiedades físicas; multimateriales capaces de responder múltiples requerimientos, de manera que se combinen las propiedades necesarias para cada solicitud: resistencia a corrosión, resistencia a desgaste, refractariedad, etc. Las posibilidades de estos materiales son dedicadas y muy diversas.

Actualmente, el compromiso de la industria, la sociedad y la ciencia en aumentar la eficiencia de los materiales en todas sus variables, principalmente económica y medioambiental, hace que se continúe investigando y desarrollando materiales con gradiente funcional o multi funcionales a distintas escalas: macro, meso, micro o nanométrica. Este desarrollo supone un análisis del ciclo de vida de los productos mayorando aquellos con menor impacto medioambiental (emisiones, energía, reciclabilidad, etc.) y menor coste económico. La industria, a su vez, reclama materiales multifuncionales que optimicen el proceso de fabricación de los productos.

Existen nuevas técnicas de procesado que abren grandes posibilidades de optimización de recursos: pulvimetalurgia, deposición de vapor física o química, cladding, fabricación aditiva, etc., para la creación de novedosas microestructuras, podríamos llamar multifuncionales, en las que cada punto tenga la respuesta solicitada, es decir, exista una geometría funcional. El estudio del entorno de la interfase de estos materiales es la llave para el diseño de los productos finales; en la cual es importante que coincidan algunas características como expansión térmica, compresibilidad y una buena mojabilidad (Heaney et al., 2003).

La compactación de polvos en matriz es una de las técnicas pulvimetalúrgicas empleadas para fabricar materiales con gradiente funcional (Mortensen y Suresh, 1995; Kieback et al., 2003, Mahamood et al., 2012). Los avances en las propiedades de los productos sinterizados pueden proceder de modificaciones en los valores de densidad/porosidad y la influencia de los poros; de modificaciones en la composición, en la microestructura o en el tamaño de grano, reduciendo su crecimiento durante la sinterización, etapa que puede incrementar las propiedades mediante distintas técnicas (Torralba y Campos, 2014). Junto a estas también tenemos, como estamos viendo, una estructuración, una ordenación de la composición en el espacio, que puede ser rentable en el futuro con un número limitado de etapas de proceso (Thomazic et al., 2010)

Esta técnica pulvimetalúrgica es muy empleada para la fabricación de un gran número de piezas de acero de diversa composición y gran consumo. Las posibilidades y calidades de los aceros sinterizados son muy numerosas, entre estos, destacan aquellos que proporcionan mejores propiedades mecánicas y mejores tolerancias dimensionales, como pueden ser los aceros sinterizados al molibdeno (Tengzelius, 2005). Además, estos aceros pueden incrementar sus interesantes propiedades con tratamientos térmicos (Torralba et al., 1995b, Candela et al., 2005).

Por ello existe la posibilidad de modificar la distribución de los elementos de los aceros donde puedan tener un mayor requerimiento, aumentando el rendimiento de la aleación; de manera que se tenga la estructuración de microestructuras y un gradiente funcional en las propiedades de los materiales obtenidos.

En este trabajo se ha diseñado la compactación de polvos en matriz de un acero sinterizado al molibdeno junto con un acero al cobre, y caracterizado las propiedades de este material compuesto estructurado. Se discuten las propiedades obtenidas al ordenar la microestructura espacialmente y dedicar las composiciones a los requerimientos.

\section{ACEROS SINTERIZADOS AL Mo CON GRADIENTE FUNCIONAL}

\subsection{Materiales}

Los materiales seleccionados para este trabajo de investigación son dos aceros con características bien diferenciadas, aunque se ha tratado que sean 
lo más compatibles posible en cuanto temperatura de sinterización y variación dimensional. Se trata de un acero con molibdeno, (polvo de $\mathrm{Fe}-3,5 \% \mathrm{Mo}$ ), aleado con cobre y grafito como material base con mayores propiedades mecánicas, al cual llamaremos material exterior (EXT). El otro acero empleado es un hierro con cobre y grafito, sin molibdeno, denominado material interior (INT). Con estos materiales se ha fabricado, según se detallará en el procedimiento experimental, un tercer acero sinterizado, $50 \%$ de EXT $+50 \%$ de INT, con gradiente de propiedades que hemos llamado FG2S. Las composiciones de estos aceros se pueden ver en la Tabla 1.

Existen referencias de las propiedades obtenidas para estas composiciones. Para el Acero con Mo EXT (Candela et al., 2001), para el acero sin molibdeno INT (Höganäs, 1997) y para la composición total del material FG2S podemos tener unos propiedades orientativas (Torralba et al., 1995a).

TABla 1. Composición química de los aceros sinterizados resultantes $(\%$ peso)

\begin{tabular}{lcccc}
\hline Material & $\mathrm{C}$ & $\mathrm{Cu}$ & $\mathrm{Mo}$ & $\mathrm{Fe}$ \\
\hline Acero con Mo: EXT & 0,7 & 3 & 3,5 & resto \\
Acero FG2S (50-50) & 0,5 & 2,5 & 1,75 & resto \\
Acero $\sin$ Mo: INT & 0,3 & 2 & - & resto \\
\hline
\end{tabular}

\subsection{Procedimiento experimental}

Con los materiales citados en el punto anterior se han fabricado las probetas por la vía pulvimetalúrgica convencional: mezcla y homogeneización de polvos, compactación en matriz flotante y sinterización en atmósfera de nitrógeno-hidrógeno.

En la primera etapa se hace la mezcla y homogeneización de los polvos metálicos en las composiciones INT y EXT que servirán para fabricar los tres tipos de materiales descritos anteriormente. La compactación de los polvos se realiza en matriz uniaxial de doble punzón a la presión de $700 \mathrm{MPa}$ (Torralba et al., 1995a; Candela et al., 2001; Garg et al., 2007). Las probetas de composición homogénea tienen un procedimiento clásico de llenado, compactación y extracción de la pieza. Las probetas FG2S, con gradiente de propiedades, sin embargo requieren otras etapas en su proceso de compactación descrito en la Fig. 1.

Se trata de una técnica de fabricación de materiales con gradiente funcional escalonado mediante compactación de polvos en matriz (Kieback et al., 2003; Mahamood et al., 2012). En este caso, el procedimiento secuencial es: llenado y precompactación a $1 \mathrm{MPa}$ nivelando superficie y cambio de material de llenado. Se puede controlar el espesor de la capa midiendo la masa que se añade a la matriz, en este caso, el material EXT se ha distribuido en la parte inferior y superior de la probeta a partes iguales.

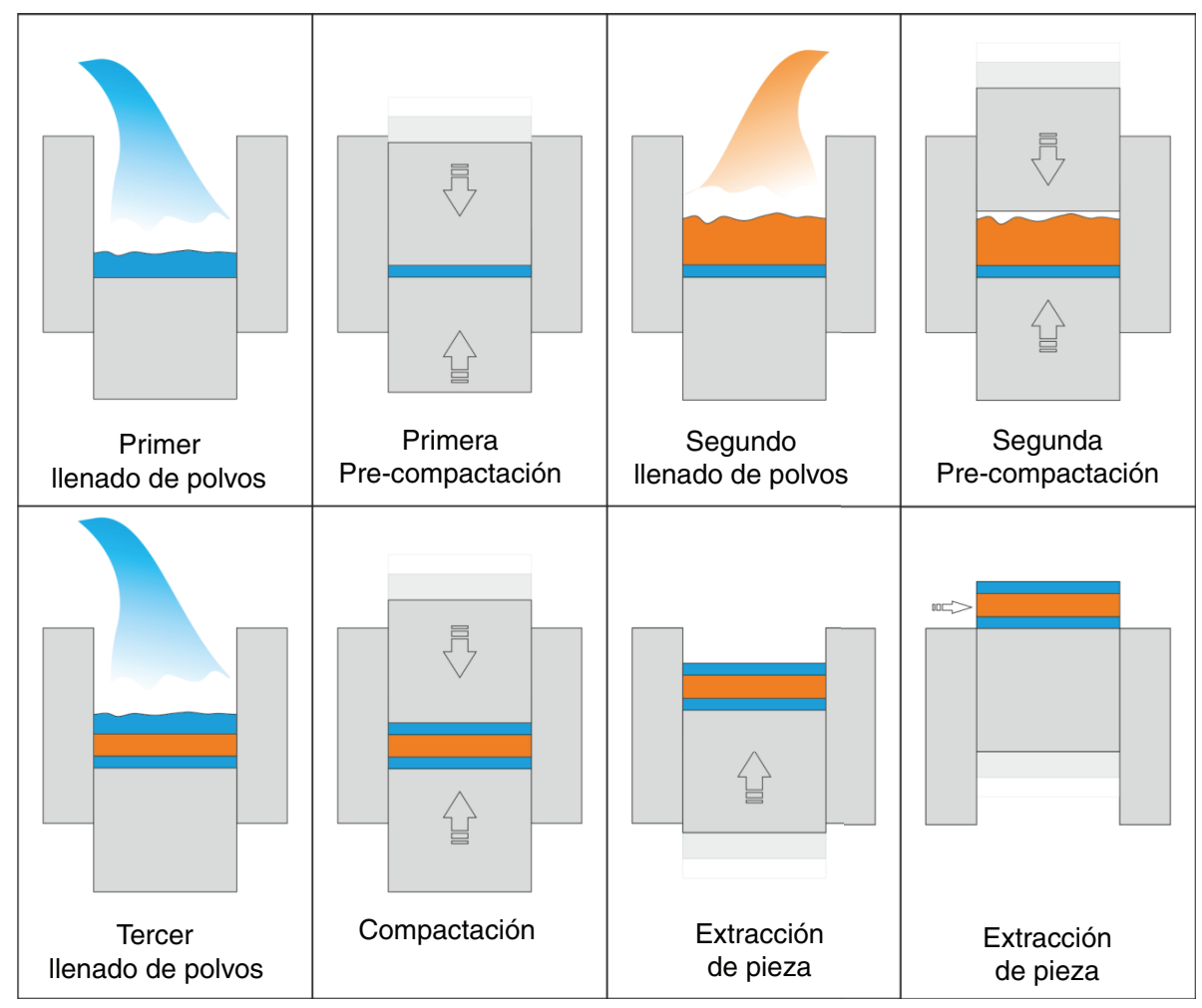

FIGURA 1. Proceso de conformación FG2S de tres capas. 
Todo el material INT se emplaza en el medio, entre las capas de material EXT, según se puede ver en la Fig. 1.

Se caracterizan las probetas en verde y se procede a la sinterización de los compactos. La sinterización se realiza en un horno tubular a la temperatura de $1120{ }^{\circ} \mathrm{C}$ durante $30 \mathrm{~min}$ en atmósfera de $\mathrm{N}_{2}-5 \mathrm{H}_{2}$.

$\mathrm{El}$ análisis químico que se ha realizado se ha centrado en constatar el gradiente de composición de las piezas. El elemento que demuestra el gradiente funcional en este estudio es el molibdeno y en él se ha realizado el análisis mediante rayos $\mathrm{X}$ con un detector incorporado en un equipo de microscopía de electrones de barrido.

Para la cuantificación de la densidad en verde, y posteriormente de la densidad del sinterizado, se procedió a la medición del espesor y anchura, con un pie de rey de $0,001 \mathrm{~mm}$ de precisión. La determinación de la masa de las probetas en verde y sinterizadas se realizó con una balanza Mettler H33AR de $0,001 \mathrm{~g}$ de precisión, para determinar las densidades de los materiales.

La determinación de la variación dimensional longitudinal (F) de las distintas probetas, se realizó mediante la aplicación de la ecuación (1):

$\mathrm{F}(\%)=(\mathrm{L} 2-\mathrm{L} 1) \cdot 100 / \mathrm{L} 1$

siendo L1 la longitud inicial de la probeta, en estado compactado (mm) y L2 la longitud final de la probeta, en estado sinterizado. La medida de esta dimensión se realizó con un calibre de $0,001 \mathrm{~mm}$ de precisión.

Los ensayos mecánicos realizados en este trabajo de investigación de los aceros al molibdeno, comprenden a los ensayos de dureza y microdureza, resistencia a tracción y alargamiento.

En el primero de ellos, la dureza, se han efectuado medidas de dureza Rockwell HRA, con un valor de la carga de $60 \mathrm{~kg}$ en todos los materiales, empleando un durómetro de la casa Hoytom. El ensayo de microdureza Vickers MHV0.1, se llevó a cabo utilizando un microdurómetro Akashi modelo MVK-E3 con cargas entre $1-1000 \mathrm{~g}$

El segundo de los ensayos mecánicos realizados fue el ensayo de tracción, que permite conocer la resistencia, límite de elasticidad, alargamiento y estricción, características básicas para evaluar la calidad de los aceros con gradiente funcional fabricados. Para el ensayo de tracción se prepararon probetas según norma ASTM B925-15 (2015), realizándose marcas separadas entre sí $35 \mathrm{~mm}$, que sirven de referencia, para determinar el alargamiento del material. Para la realización del ensayo se empleó una máquina de tracción universal de $10 \mathrm{t}$ a velocidad constante de $10 \mathrm{~kg} \cdot \mathrm{s}^{-1}$ de aplicación de la carga.

Finalmente se determinan las propiedades resultantes del proceso de fabricación: densidad, variación dimensional, durezas, resistencia a tracción, alargamiento y metalografía: óptica (LOM) y electrónica de barrido de electrones (SEM).

\section{RESULTADOS Y DISCUSIÓN}

Los resultados obtenidos de los materiales con fase homogénea se corresponden con los presentados en estudios previos (Höganäs, 1997; Candela et al., 2001). La combinación de fases homogéneas en el material FG2S tiene una interfase discreta, como se puede ver en la Fig. 2, donde puede apreciarse la diferencia de composición elemental en las distintas zonas del material FG2S fabricado y en el que se logra una completa unión metalúrgica. Las composiciones elegidas para el exterior e interior del acero con gradiente funcional FG2S, son compatibles y permiten que tras el proceso de compactación en matriz y posterior sinterización, no se presenten grietas ni fisuras producto de las diferencias en los valores de variación dimensional que se producen en las distintas zonas, como se irá confirmando con el análisis de los resultados que se expondrán a continuación.

\subsection{Densidad}

El material FG2S presenta una buena densificación debida a la sinterización en fase líquida que proporciona el cobre. Las densidades de la Fig. 3 muestran valores diferentes para el material más blando del interior INT y el duro EXT como se produce en materiales compactados a la misma presión. El material FG2S tiene una densidad total similar al material duro. Sin embargo se puede observar el gradiente de porosidad en la Fig. 4, donde se aprecia que la porosidad es mayor en las zonas del material EXT (Arriba y Abajo) y menor para el INT. Podemos correlacionar con el análisis de imagen este gradiente de porosidad con un gradiente de densidades resultantes de la variación de esfuerzos que se producen durante la compactación por la configuración de las fases duras y blandas en las piezas (Thomazic et al., 2010).

\subsection{Variación dimensional}

Una de las variables fundamentales en este proceso de fabricación es la variación dimensional que se produce en los materiales durante su sinterización. Hay que recordar que en este proceso, estamos sinterizando un material que tiene diferentes composiciones químicas en distintas áreas. Eso provocará una situación compleja, ya que la variación dimensional que experimentará será diferente en cada una de esas zonas.

La variación dimensional de los materiales responde a las composiciones de los materiales 


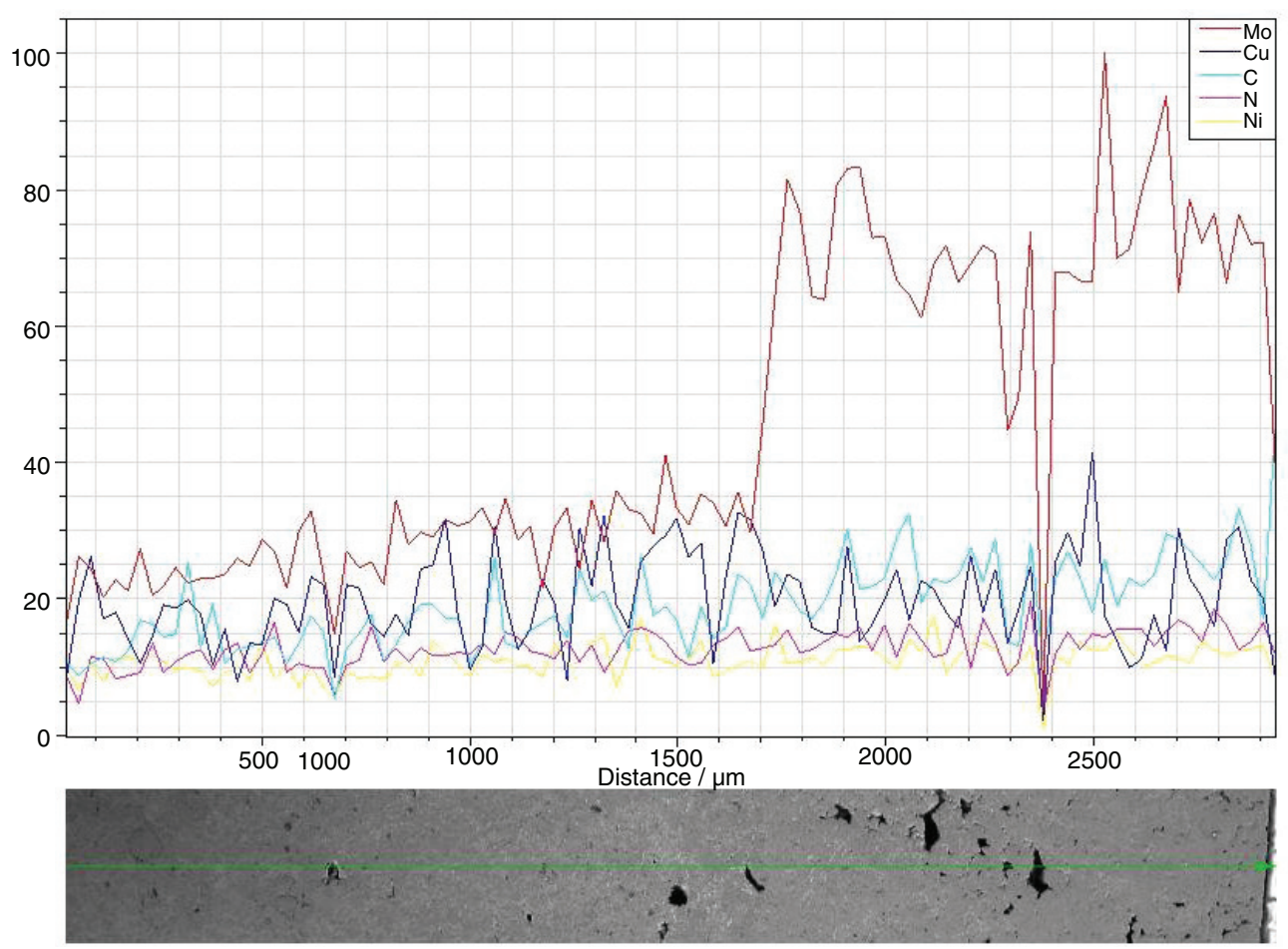

FIgURA 2. Gradiente de composición de la interfase FG2S.

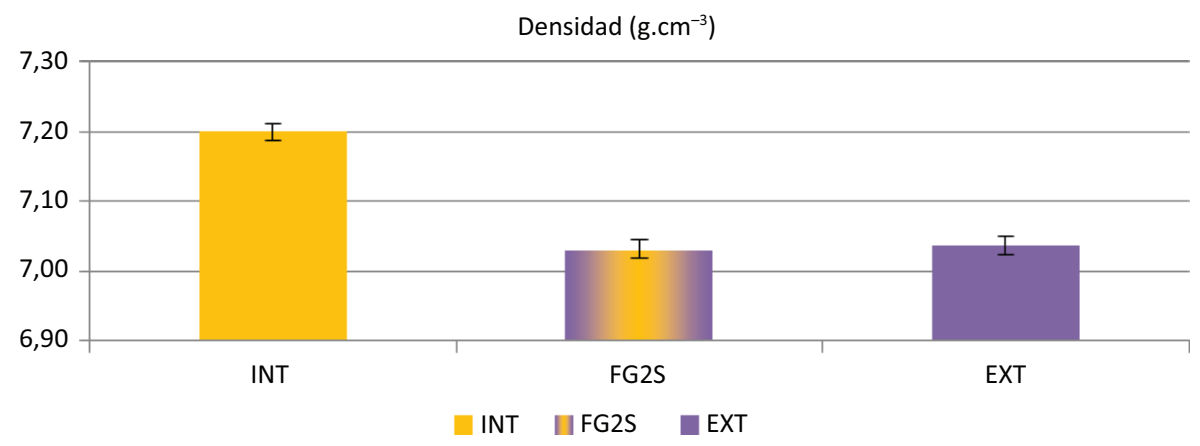

Figura 3. Densidad de los materiales.

homogéneos: mayor variación para el hierro con cobre debido al fenómeno de hinchamiento que produce este elemento y menor variación del acero con $\mathrm{Mo} \mathrm{y} \mathrm{Cu}$ dado que la mayor presencia de $\mathrm{C}$, junto con el Mo aumenta el ángulo de diedro y facilita la difusión de cobre. Para el material graduado FG2S la variación dimensional es intermedia entre los valoras obtenidos con los dos materiales homogéneos, resultando próxima al $0,5 \%$ (Fig. 5). A causa de esta diferencia de dilataciones de los materiales se puede decir que existe un estado tensional residual en la interfase que, debido a la configuración de las capas, no ha producido distorsiones; de manera que el material interior tracciona el material exterior (Suresh y Mortensen, 1997).

\subsection{Dureza}

Los valores de la dureza que se pueden ver en la Fig. 6 son de la superficies superior e inferior de las probetas, coherentes con los resultados existentes, el 3,5\% de Mo junto con al 0,7 de C proporciona una dureza mayor que la del hierro con 0,3 de $\mathrm{C}$; ambos con un $3 \%$ y un $2 \%$ de $\mathrm{Cu}$, respectivamente.

En el material FG2S, se mantiene la dureza del material EXT. Esta es una de las principales aplicaciones de los materiales con gradiente funcional (Bever y Duwez, 1972). En este caso, una distribución de durezas en los puntos que demandan mayores durezas, en el exterior, según se puede observar en la Fig. 7. Estas durezas responden al gradiente de 


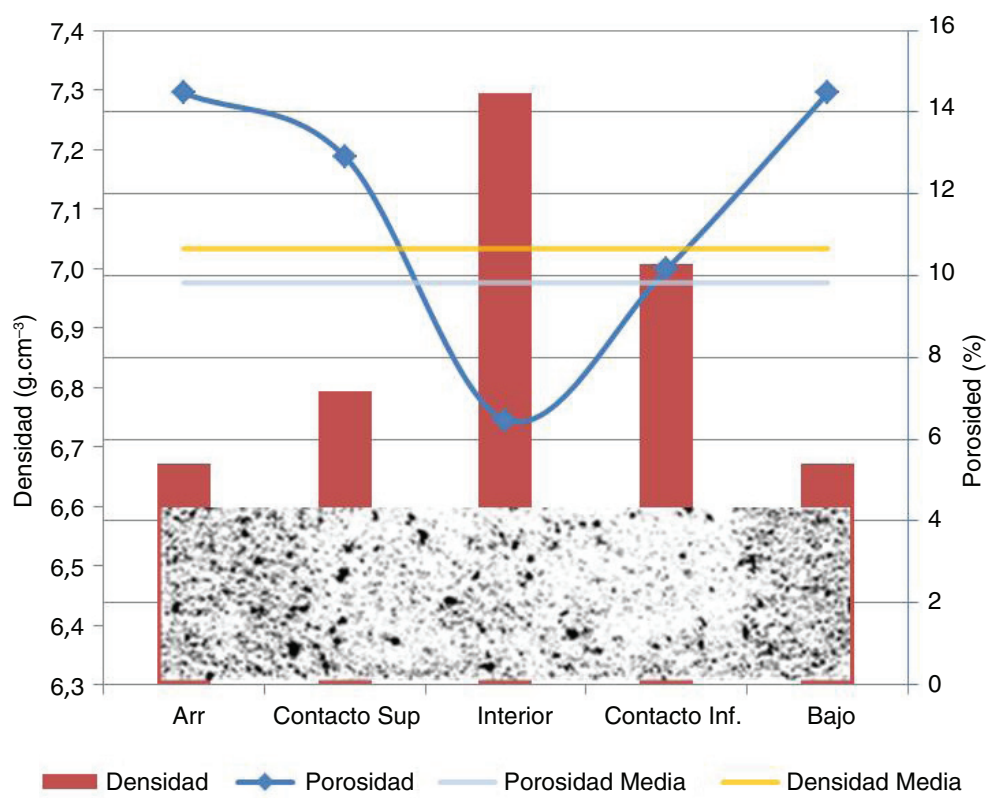

FIGURA 4. Gradiente de porosidad y densidad correlacionada.

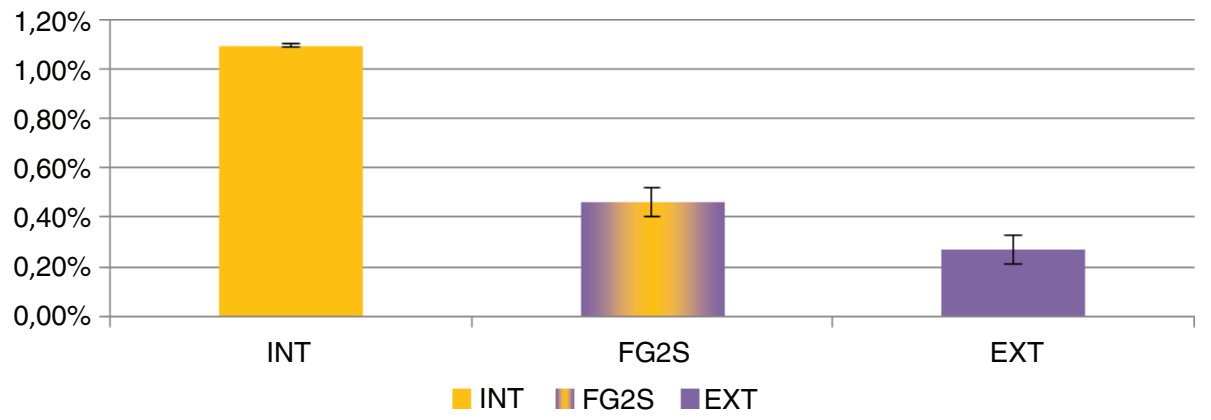

Figura 5. Variación dimensional tras sinterización.

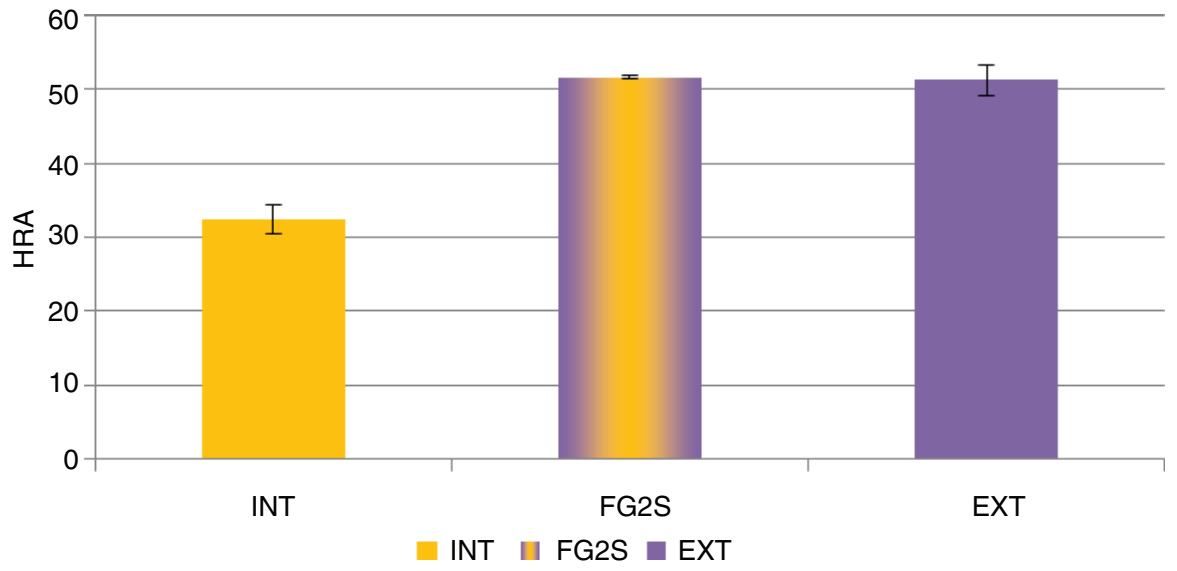

FIgURA 6. Dureza de los aceros sinterizados con gradiente funcional y homogéneo. 
fases: bainitas, perlitas y ferritas como se analizará más adelante.

\subsection{Propiedades mecánicas}

Las resistencias de los aceros sinterizados homogéneos estudiados coinciden con los resultados recogidos en la literatura para las composiciones

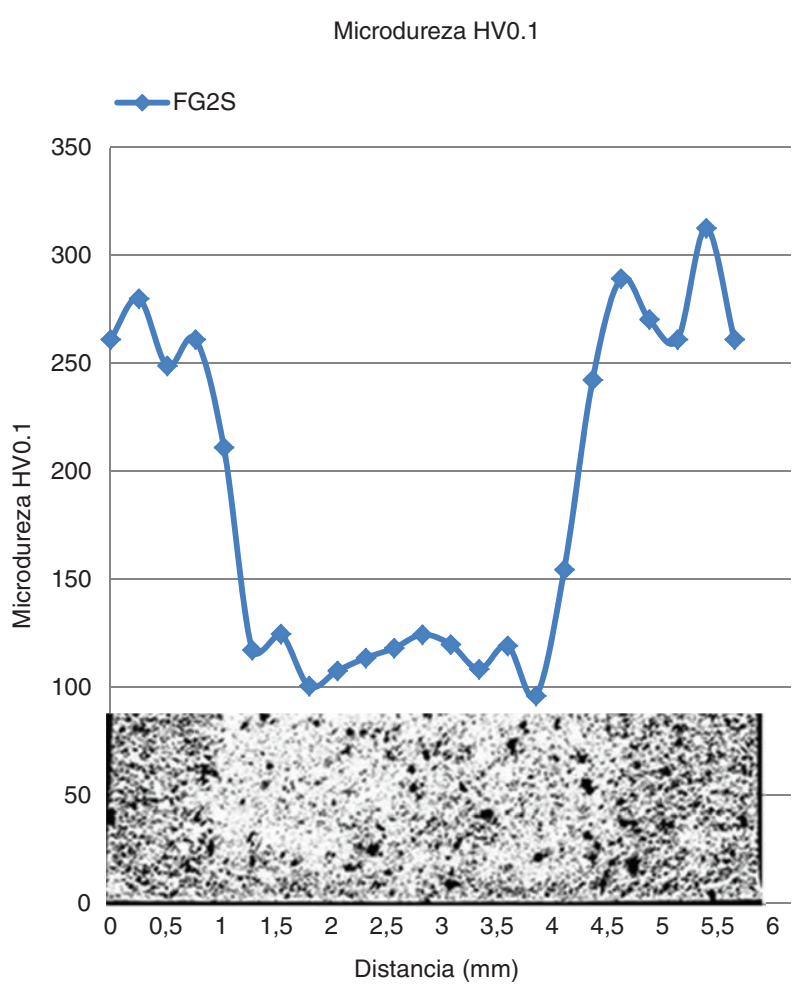

FIGURA 7. Gradiente de microdurezas en la sección del acero FG2S. resultantes de los materiales (Torralba et al., 1995a; Höganäs 1997; Candela et al., 2001)

Se puede ver en la Fig. 8 que debido a la estructura en el material FG2S, con una configuración diseñada en tres capas, resultan unos valores de resistencia a tracción que se aproxima a la regla de la mezclas de los materiales compuestos. Se observa en la misma figura que el límite elástico es relativamente superior en el material confeccionado FG2S, proporcionando una rigidez mayor.

El alargamiento del material EXT coincide con el de FG2S $(1,4 \%)$, menor que el alargamiento del material INT de la Fig. 9. El estado tensional resultante de la sinterización de materiales con diferente variación dimensional del FG2S influye en las propiedades mecánicas finales; dado que el material con mayor variación dimensional resultaría en compresión y en tracción el de menor dilatación de sinterización. Es decir, el material en tracción tendría así una capacidad de deformación menor que si no estuviera tensionado. Esto puede justificar el aumento de la rigidez y del límite elástico en relación con las fases homogéneas.

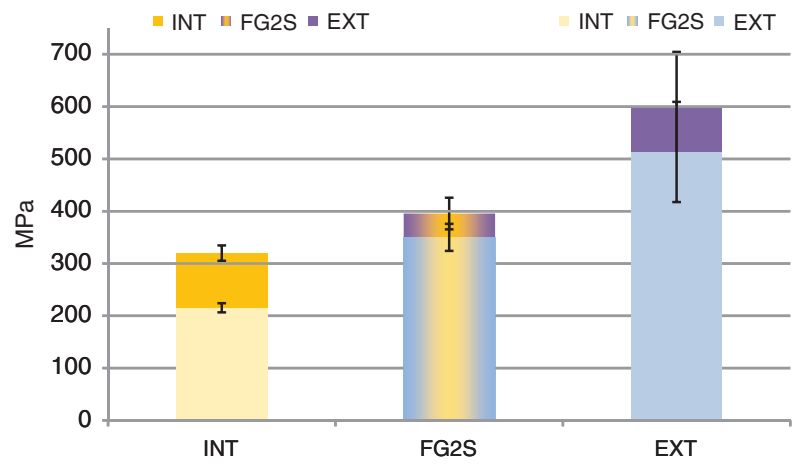

FIgURA 8. Resistencia a tracción y límite elástico de los aceros sinterizados.

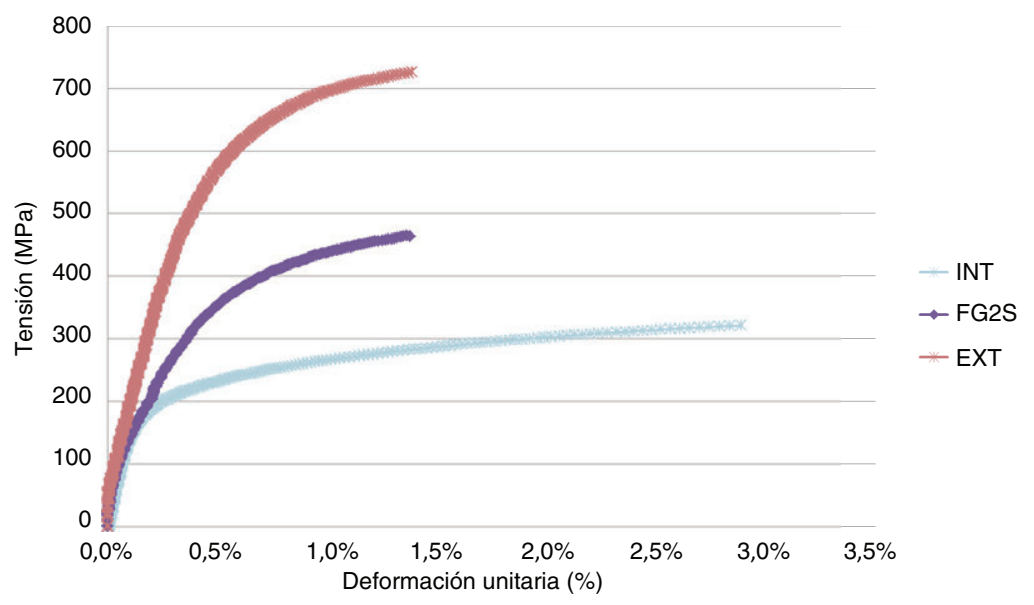

Figura 9. Curvas de tensión-deformación de los aceros sinterizados. 


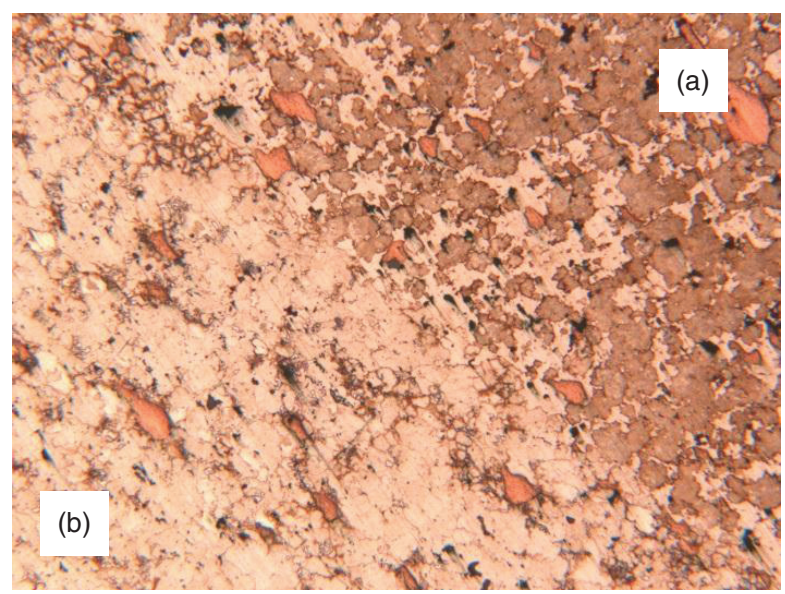

Figura 10. Microestructura del material FG2S: a) Zona exterior: bainitas y perlitas finas con áreas de $\mathrm{Cu}$, b) Zona interior: Ferritas ricas en $\mathrm{Cu}$, perlita y áreas de $\mathrm{Cu}$. Atacada con nital. $250 \mathrm{X}$.

\subsection{Análisis microestructural}

En cuanto al análisis metalográfico del acero con gradiente funcional fabricado FG2S, se puede observar que se consigue el efecto buscado de tener perfectamente diferenciadas las zonas con diferente estructura. En la Fig. 10 se muestra la microestructura correspondiente a las dos zonas de diferente composición. La zona exterior correspondiente a la composición EXT constituida por bainitas y perlitas finas con áreas de $\mathrm{Cu}$, y la zona interior correspondiente a bajo contenido en carbono y $\mathrm{Cu}$, constituida por ferritas ricas en $\mathrm{Cu}$, perlitas y áreas de $\mathrm{Cu}$ libre, lo que justifica los valores de los resultados obtenidos en cuanto a comportamiento mecánico de este acero con gradiente funcional FG2S fabricado en configuración de tres capas.

$\mathrm{Si}$ analizamos en la fractura del acero sinterizado FG2S se puede apreciar que muestra comportamientos diferenciados en las zonas de distinta composición: rotura frágil en la zona correspondiente (a)

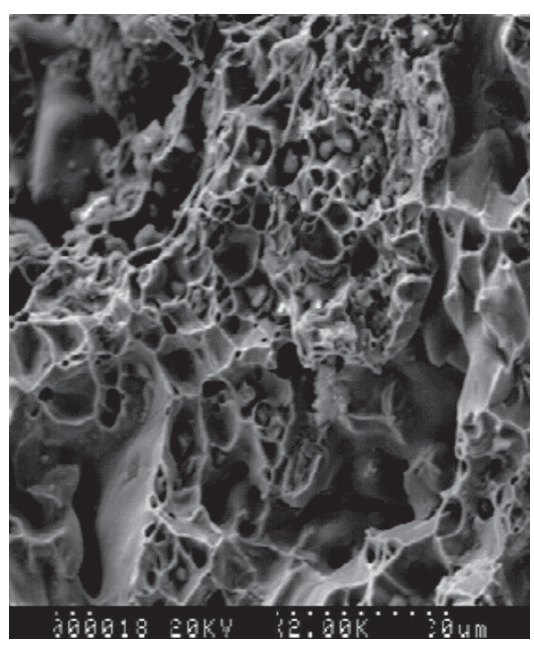

(b)

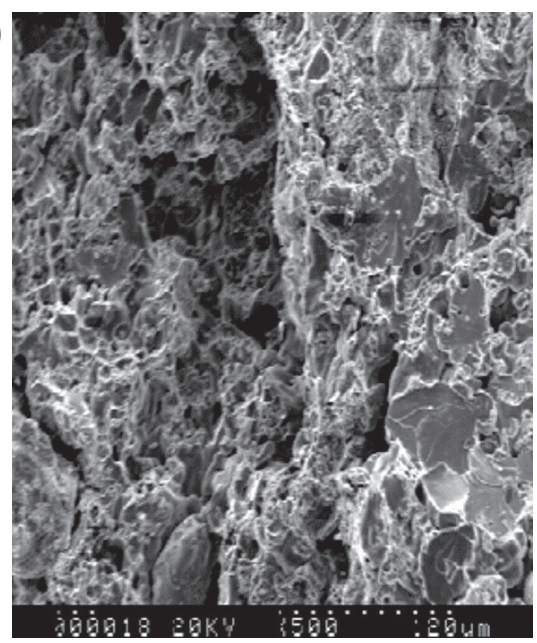

(c)

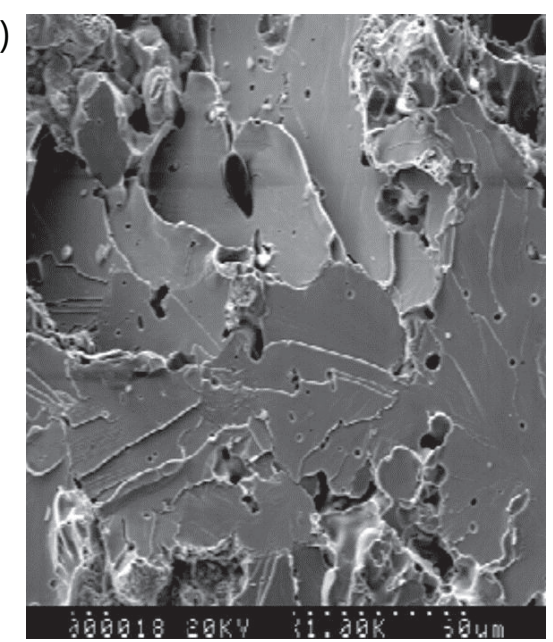

FIGURA 11. a) fractura dúctil con cuellos en la zona del acero INT., b) fractura dúctil - frágil en la interfase del acero con gradiente funcional FG2S y c) fractura frágil transgranular en la zona de composición EXT. 


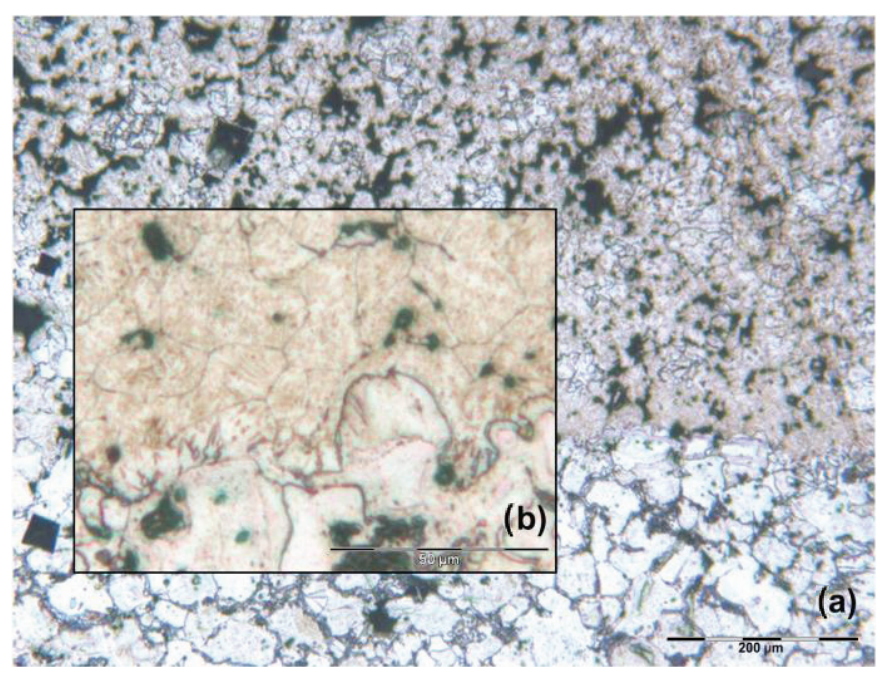

Figura 12. Interfase de FG2S: a) 100X aumentos, b) 500X aumentos.

al exterior donde se concentran el Mo y el C y por tanto es la zona de mayor dureza y fractura dúctil en la zona de menor contenido en $\mathrm{C} \mathrm{y} \mathrm{Cu}$ correspondiente al interior.

En la Fig. 11c se puede ver una rotura frágil transgranular en (Fig. 11c), junto con unos huecos en menor cantidad de la deformación dúctil del acero sinterizado EXT (Candela et al., 1999). La fractura dúctil de acero INT se puede observar en la Fig. 11a completamente diferente a la del material duro. Una combinación de las fracturas anteriores se localizan en el acero FG2S (Fig. 11b) como corresponde a las fases que la componen, a la izquierda el acero INT y a la derecha el más duro EXT. En esta misma imagen se puede apreciar cómo se produce un escalón provocado por la descohesión de las fases durante la rotura ya que como se puede observar en la Fig. 12, la interfase no presenta fisuras debidas al proceso de sinterización.

\section{CONCLUSIONES}

La combinación FG2S de dos composiciones de acero en una geometría definida ofrece propiedades con gradiente funcional. Por tanto podemos concluir que el proceso de compactación en matriz diseñado es válido para la fabricación de aceros sinterizados con gradiente funcional en una geometría definida. Estudiando la compatibilidad de las composiciones se pueden producir piezas con propiedades que satisfagan múltiples requerimientos.

En este estudio, la combinación de los materiales dedicados es viable y destaca porque:

- La unión metalúrgica de las composiciones de acero empleadas en las distintas capas INT y EXT (al molibdeno) con cobre es buena, sin fisuras durante el proceso de sinterización debido a diferencias en la variación dimensional.

- Las piezas no presentan distorsión o deformación tras la sinterización. El diseño de tres capas proporciona simetría en el plano con mayor área. Aunque sí se puede entender que existen tensiones residuales derivadas de las diferencia de variación dimensional durante la sinterización.

- Se observa una variación gradual de la resistencia mecánica a lo largo del material. La composición más resistente resulta menos densa que la composición más blanda que se compacta y se densifica en mayor medida.

- Se alcanzan durezas, que ofrece el material estructurado, un $25 \%$ mayores que la misma composición global equivalente, es decir, comparado con los materiales que emplean los mismos aleantes sin estructurar la pieza.

- Los resultados de resistencia del material ordenado ofrecen una mayor resistencia y rigidez similar a la que se obtiene de la regla de mezclas de los materiales compuestos. Y aumenta el límite elástico en relación a la resistencia a tracción.

\section{REFERENCIAS}

ASTM B925-15 (2015). Standard Practices for Production and Preparation of Powder Metallurgy (PM) Test Specimens, ASTM International, West Conshohocken, PA, California, USA.

Bever, M., Duwez, P. (1972). Gradients in Composite Materials. Mater. Sci. Eng. 10, 1-8. http://dx.doi.org/ 10.1016/0025-5416(72)90059-6.

Candela, N., Velasco, F., Torralba, J.M. (1999). Fracture mechanisms in sintered steels with $3.5 \%$ (Wt.) Mo. Mat. Sci. Eng. A-Struct. 259 (1), 98-104. http://dx.doi.org/10.1016/ S0921-5093(98)00865-X 
Candela, N., Antón, N., Gordo, E., Velasco, F., Torralba, J.M. (2001). Mechanical properties and wear behaviour of nitrided Fe-3.5Mo base sintered steels. Mater. Sci. Tech. 17 (3), 309-314. http://dx.doi.org/10.1179/026708301773002518.

Candela, N., Velasco, F., Martínez, M.A., Torralba, J.M. (2005). Influence of microstructure on mechanical properties of molybdenum alloyed P/M steels. J. Mater. Process. Tech. 168 (3), 505-510. http://dx.doi.org/10.1016/j. jmatprotec.2004.02.066.

Garg, P., Park, S., German, R.M. (2007). Effect of die compaction pressure on densification behavior of molybdenum powders. Int. J. Refract. Met. H. 25 (1), 16-24. http:// dx.doi.org/10.1016/j.ijrmhm.2005.10.014

Heaney, D., Suri, P., German, R.M. (2003). Defect-free sintering of two material powder injection molded components Part I. Experimental investigations. J. Mater. Sci. 38 (24), 4869-4874. http://dx.doi.org/10.1023/ B:JMSC.0000004407.63082.f1.

Höganäs, A.B. (1997). Handbook for Sintered Components. Design and Mechanical Properties, Chapter 9: Sintered Iron-based Materials, pp. 57-58-61

Kieback, B., Neubrand, A., Riedel, H. (2003). Processing techniques for functionally graded materials. Mat. Sci. Eng. A-Struct. 362 (1-2), 81-106. http://dx.doi.org/10.1016/ S0921-5093(03)00578-1.

Mahamood, R.M., Akinlabi, E.T., Shukla, M. Pityana, S. (2012). Functionally Graded Material: An Overview. Proceedings of the World Congress on Engineering (WCE 2012), London, England.

Mortensen, A., Suresh, S. (1995). Functionally graded metals and metal-ceramic composites: Part 1 Processing. Int.
Mater. Rev. 40 (6), 239-265. http://dx.doi.org/10.1179/ imr.1995.40.6.239.

Suresh, S., Mortensen, A. (1997). Functionally graded metals and metal-ceramic composites: Part 2 Thermomechanical behaviour. Int. Mater. Rev. 42 (3), 85-116. http://dx.doi. org/10.1179/imr.1997.42.3.85.

Tengzelius, J. (2005). Advances in Steel Powders for High Performance PM Parts, Presented at PMAsia2005, Shanghai. https://www.researchgate.net/profile/Jan_Tengzelius/ publication/265243729_ADVANCES_IN_STEEL_POWDERS_FOR_HIGH_PERFORMANCE_PM_PARTS/ links/557ffcbb08aeb61eae26ee77.pdf

Thomazic, A., Guennec, Y.L., Kamdem, Y., Pascal, C., Chaix, J.M., Doremus, P., Imbault, D., Bouvard, D., Doré, F. (2010). Fabrication of bimaterial components by conventional powder metallurgy. International Powder Metallurgy World Congress \& Exhibition (PM2010), Florence, Italy.

Torralba, J., Candela, N., Ruiz-Roman, J.M., Cambronero, L., Ruiz-Prieto, J.M. (1995a). Caracterización de los aceros sinterizados obtenidos a partir de polvos prealeados Fe-1, 5\% Mo. Rev. Metal. 31 (1), 14-22. http://dx.doi. org/10.3989/revmetalm.1995.v31.i1.

Torralba, J., Candela, N., Velasco, F., Cambronero, L., RuizPrieto, J.M. (1995b). Tratamientos térmicos de los aceros sinterizados obtenidos a partir de polvos prealeados Fe-1, 5\% Mo. Rev. Metal. 31 (2), 71-77. http://dx.doi. org/10.3989/revmetalm.1995.v31.i2.

Torralba, J.M., Campos, M. (2014). Toward high performance in powder metallurgy. Rev. Metal. 50 (2), e017. http://dx.doi. org/10.3989/revmetalm.017. 